\title{
Coloration and structure behavior after silver and copper nanoparticles formation in soda lime glass
}

\section{Farb- und Strukturverhalten von Kalk-Natron-Glas nach der Bildung von Silber- und Kupfernanopartikeln}

\author{
A. Chorfa ${ }^{1,2}$, N. Belkhir ${ }^{1}$, A. Belbali ${ }^{1}$, F. Rubio ${ }^{3}$, J. Rubio $^{3}$
}

In this work, an ion exchange non-conventional process commonly called ancient painting process without using molten salts is used as a method for glass coloring. In this process of coloration, a mixture of metallic particles with kaolin and arabic adhesive is applied on the soda lime glass surface. Afterwards, the painted glass is submitted to the heat treatment to obtain the color. A comparative study is carried out in order to investigate the diffusion of metallic nanoparticles (silver and copper) in soda lime glasses depending on several parameters as the concentration and composition of stain spread on glass, and the heat treatment temperature. Characterization of the composition and structure of the painted glasses is performed by UV-visible spectroscopy. In addition, nanoindentation and scanning electron microscopy (SEM) with energy dispersive X-ray analysis (EDS) techniques are used to determine the penetration of metallic nanoparticles into glass. The obtained result shows that the coloration of painted glass differs and depends on the concentration and the kind of the mixture of the paw. Moreover, it was found that the effect of the heat treatment temperature is primordial on the formation and diffusion of metallic nanoparticles.

Keywords: Metallic nanoparticles / glass / ion exchange / spectroscopy

Schlüsselwörter: Metallische Nanopartikel / Glas / lonenaustausch / Spektroskopie

\section{Introduction}

Metallic nanoparticles are indeed tools tailored not only by their easy synthesis but also by their large capacity functionalizing surface [1]. However, the glasses containing metallic nanoparticles have attracted great interest and research especially in the last years due to their large application in: Photonics for their linear and non-

1 Institute of optics and precision mechanics, Ferhat Abbas Sétif University, El Bez Campus, SÉTIF 19000, ALGERIA

2 Research Unit, emerging materials, Ferhat Abbas Sétif University, El Bez Campus, SÉTIF 19000, ALGERIA

3 Glass and ceramic institute (icv) CSIC, Campus de Cantoblanco, 28049 MADRID, SPAIN linear optical properties, biomedical, biological, and decoration [2-7]. Introducing silver and copper nanoparticles into glasses has been used for coloring glasses and recently for fabricating optical devices [8]. It has been used for decorative purposes for a very long time because of its colors, which can be yellow or orange, red or amber, due to the scattered particles of cuprous or cupric oxides as well as copper or silver metallic

Corresponding author: N. Belkhir, Institute of optics and precision mechanics, Ferhat Abbas Sétif University, El Bez Campus, SÉTIF 19000, ALGERIA, E-Mail: belkhir_nabil@univ-setif.dz 
nanoclusters. In the last few years, these glasses have attracted attention for the blue-green luminescence properties of copper-glasses, useful for laser technology [9]. An enormous interest has grown in the development of new preparation methods of these metal nanoclusters composite glasses. Such glasses have been recently prepared by ion implantation, radiation using laser, X-ray, or $\gamma$-ray, and sol gel technique [10-12]. However, the mainly used method is still the ion exchange, which provides an easy and efficient formation of metallic nanoparticles in glasses structure [13]. The ion exchange process in soda lime glass has generated considerable interest, by its consideration as a prime technique for integrated optical device applications. In addition, it is one of the simplest and most effective methods of making optical waveguides and gradient index lenses in glasses [14].

For glasses coloration, artists have used another process, which is the classic staining method [1517]. It is a non-conventional method where the ion exchange is accomplished without molten salts use. It is applied on the glasses surface, where the metallic element is mixed with ocher and clay then diluted with various organic carriers (glue, wine vinegar, spirit of wine, etc.) that act as binders [15].

In a previous work, this technique was used for the staining of two types of glass, soda lime and borosilicate glass [18]. It has been shown that the distribution and size of the silver nanoparticles, formed in borosilicate glass, are less important than those formed in soda lime glass. This was due to the presence of high levels of alkaline in it and the length of the heat treatment, which affect slightly the silver depth distribution. Nevertheless, they affect the size of formed nanoparticles and therefore affect the color of painted glass.

In this work, the classic method was used for the staining of soda lime glass by unary or sequential ion exchange (silver-copper and copper-silver). The objective is to clarify the influence of the complex mechanism of coloration generated by the formation of copper and silver nanoparticles on changing the structure of painted glass.

\section{Experimental study}

The studied glass is a soda lime glass whose composition was determined by X-ray fluorescence technique, Table 1. Transition temperature $\left(T_{\mathrm{g}}\right)$ of the used glass obtained by differential thermal analysis (DTA) is about $595{ }^{\circ} \mathrm{C}$.

The used samples were of parallelepiped shape with dimensions of $30 \mathrm{~mm} \times 30 \mathrm{~mm} \times 5 \mathrm{~mm}$ (length $\mathrm{x}$ width $\mathrm{x}$ thickness). A copper and silver stain was prepared, and spread on the surface of soda lime glasses using a small brush. For each sample, the mixture was prepared using $40 \mathrm{mg}$ of kaolin, two drops of Arabic adhesive and copper nitrate, copper sulfate and silver nitrate. Three different compositions of these metallic components were used. Combinations are designed respectively by $\mathrm{N}, \mathrm{S}$ and $\mathrm{Ag}$ and are name as follows:

1. N1/S1/Ag1:10 mg of copper nitrate or copper sulfate or silver nitrate;

2. N2/S2/Ag2: $15 \mathrm{mg}$ of copper nitrate/copper sulfate/silver nitrate;

3. N3/S3/Ag3: $20 \mathrm{mg}$ of copper nitrate / copper sulfate / silver nitrate.

The substrates are then heat treated at a temperature usually lower than the glass transition temperature, in order to obtain copper/silver diffusion into the glass structure and the development of the red/yellow color $[16,17]$. Thus, samples were heated in air for 2 hours at different temperatures $\left(580{ }^{\circ} \mathrm{C}, 480{ }^{\circ} \mathrm{C}, 350{ }^{\circ} \mathrm{C}\right)$. The heating rate was $2^{\circ} \mathrm{C} \cdot \mathrm{min}^{-1}$, and the cooling rate was $1^{\circ} \mathrm{C} \cdot \mathrm{min}^{-1}$. At the end, the painted glasses surfaces were very well cleaned before characterization.

The optical transmission spectra of prepared samples were measured using a UV-visible spectrophotometer (Perkin-Elmer Lambda model 40) with flat soda-lime glass as a reference. Measurements were performed at room temperature in the wavelengths range of $300 \mathrm{~nm}$ to $1100 \mathrm{~nm}$. The penetration depth of the cooper and silver nanoparticles in the painted glass was determined via a field emission scanning electron microscopy (FESEM) with energy dispersive X-ray spectroscopy (EDS) using a Hitachi S-4700 equipment working at $20 \mathrm{kV}$.

Table 1. Chemical composition of the studied glass.

\begin{tabular}{lllllllll}
\hline Content (Wt. \%) & $\mathrm{SiO}_{2}$ & $\mathrm{Al}_{2} \mathrm{O}_{3}$ & $\mathrm{Na}_{2} \mathrm{O}$ & $\mathrm{K}_{2} \mathrm{O}$ & $\mathrm{CaO}$ & $\mathrm{MgO}$ & $\mathrm{Fe}_{2} \mathrm{O}_{3}$ & Others $^{*}$ \\
\hline Soda lime & 72.40 & 1.26 & 13.40 & 0.24 & 8.53 & 3.95 & 0.16 & 0.06 \\
\hline
\end{tabular}

* Others: $\mathrm{P}_{2} \mathrm{O}_{5}, \mathrm{TiO}_{2}, \mathrm{ZrO}_{2}$. 
The equipment used for the nano indentation test (NanoTest Micro Materials Ltd) is a static system that functions with a horizontal loading mechanism. It is characterized by a loading range $(0 \mathrm{mN}-500 \mathrm{mN})$ with a force and a displacement resolutions $0.1 \mathrm{mN}$ and $0.1 \mathrm{~nm}$ respectively. The equipment is well protected against significant thermal drift, ambient air flow and acoustic disturbance. It is equipped with a Nano-K bench top vibration isolation platform.

\section{Results}

The absorption spectra of the studied glasses shows that unlike raw glass S0, the painted glasses with copper mixture have an apparent absorption in the visible range, characterized by the appearance of an absorption peak near the $570 \mathrm{~nm}$ wavelength. It is illustrated that the absorption peak is more intense as the concentration of the copper paste spread on the glasses is high, and the coloration of the glasses painted with the copper sulfate is more intense than those painted with copper nitrate $78 \%$ and $52 \%$ respectively. It is noted that the spectrum of the glass painted with copper nitrate at low concentration (N1) is characterized by the absence of a peak near the wavelength $570 \mathrm{~nm}$ showing the non-formation of copper nanoparticles in this glass structure, Figure 1.

The treatment temperature plays an important role on the transmittance of soda lime glasses. Indeed, the highest values of transmittance are obtained when glasses painted with copper nitrate or copper sulfate are

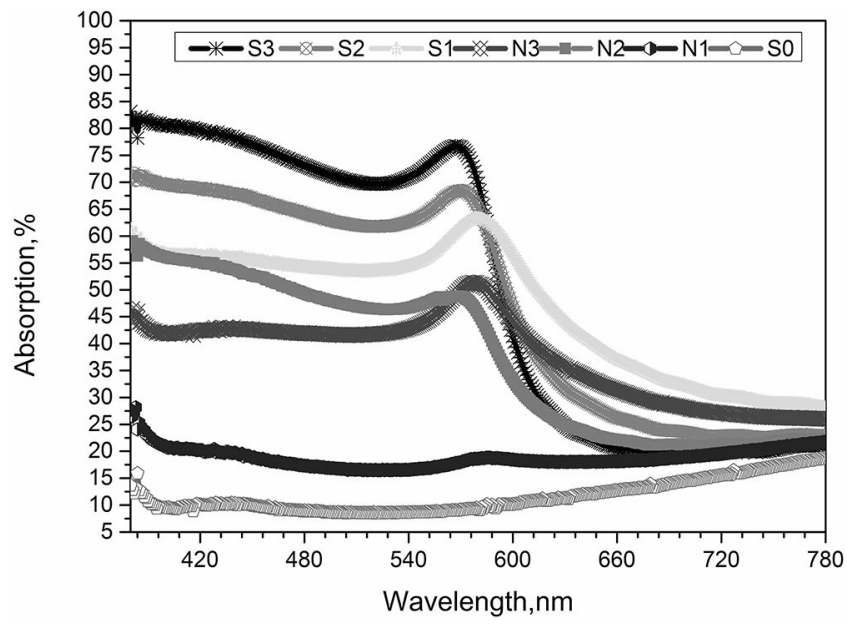

Figure 1. UV-visible absorption spectra of the copper-stained soda lime glasses treated at $580{ }^{\circ} \mathrm{C}$, vs. stain concentration, stain composition, (SO: raw glass). heat treated at $350{ }^{\circ} \mathrm{C}$. No peaks are observed for this treatment temperature. However, when the heat treatment temperature is increased up to $480{ }^{\circ} \mathrm{C}$ a peak located close to $570 \mathrm{~nm}$ begins to appear. This peak increases its intensity when the temperature is raised up to $580{ }^{\circ} \mathrm{C}$, Figure 2 .

The indentation curves of painted glasses have shown that the maximum penetration depth of painted glasses with copper sulfate (S3) is higher than that of glasses painted with copper nitrate (N3). The latter exhibit a similar behavior as the raw glasses (S0), Figure 3.

Furthermore, the slope of the unloading beginning of the indentation curve, characterizing the elastic modulus of the studied glasses, depends on the spread paste composition [19]. This reflects the elastic modulus found about $78.43 \mathrm{GPa}, 76.33 \mathrm{GPa}$,
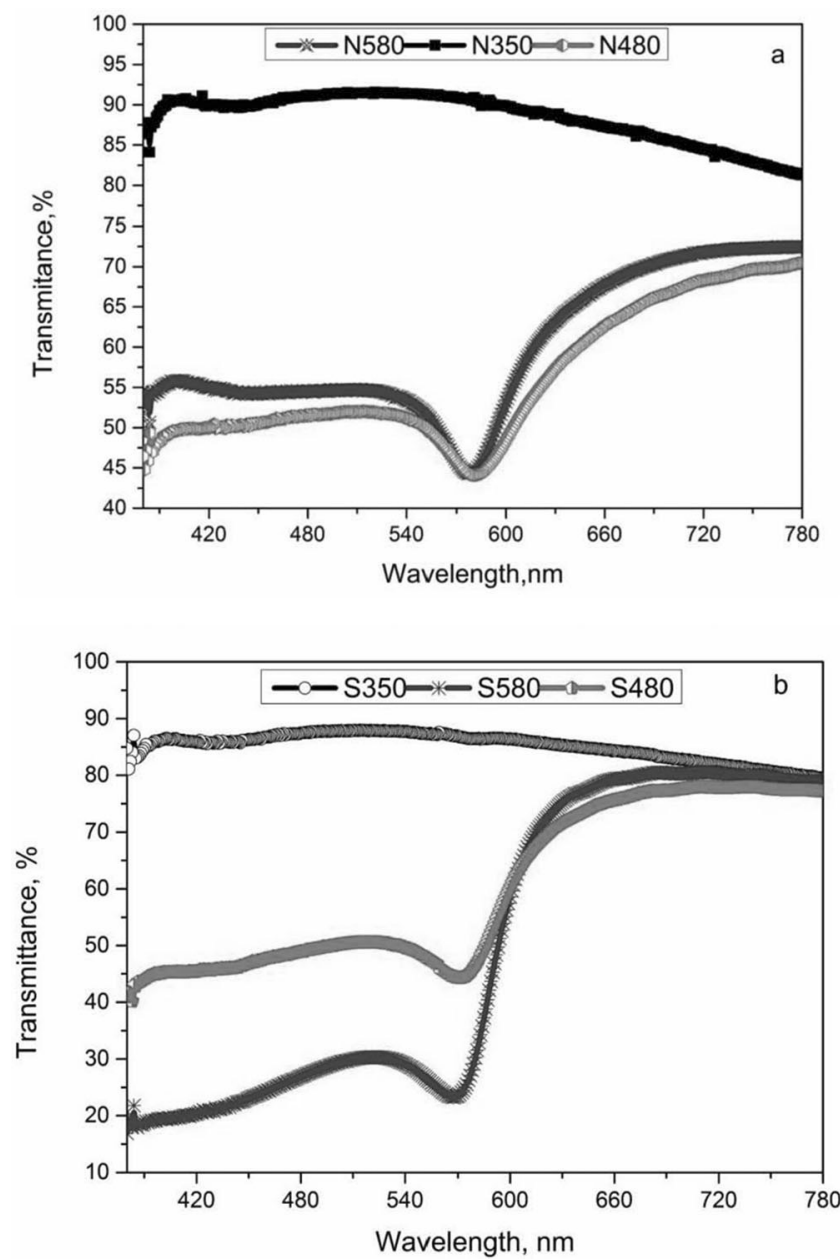

Figure 2. UV-visible transmission spectra of the copper-stained soda lime glasses, vs. heat treatment temperature and stain concentration, a) copper nitrate stain, b) copper sulfate stain. 


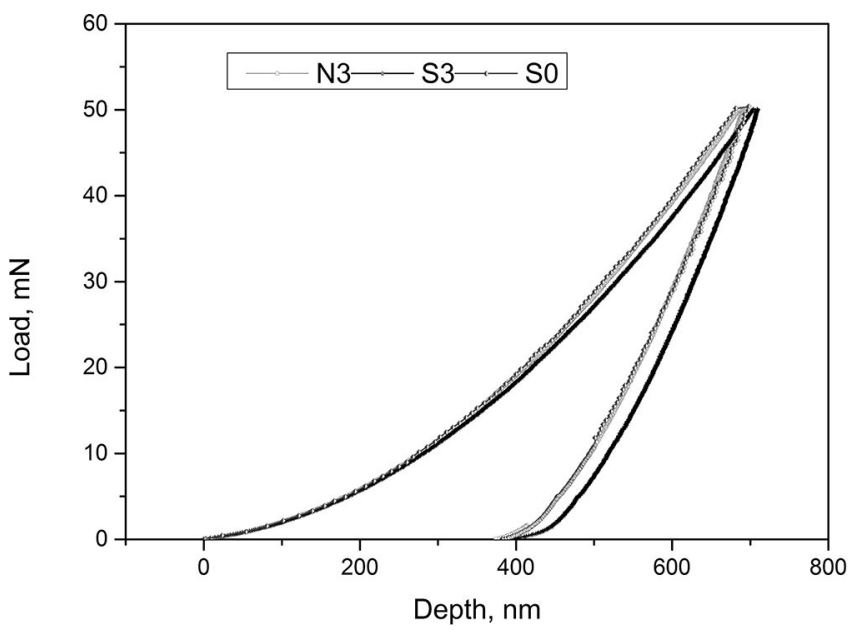

Figure 3. Indentation load-displacement curve for stained soda lime glass (S3, N3) and raw glass (S0).

$75.69 \mathrm{GPa}$, for the raw glass (S0), copper nitrateglass (N3), and copper sulfate-glass (S3) respectively. This indicates that the copper nanoparticles diffusion in the glass reduces its stiffness.

Glasses painted with silver nitrate presented a more intense absorption than glasses painted with copper nitrate. On the other hand, silver glass (Ag3) present an absorption peak located at $420 \mathrm{~nm}$ while that of copper nitrate glass $(\mathrm{N} 3)$ is at $570 \mathrm{~nm}$. This difference in absorption indicates that greater opacity is obtained for silver glasses than for copper ones, Figure 4.

The diffusion of silver into the soda lime glass is greater than copper sulfate and copper nitrate, Fig-

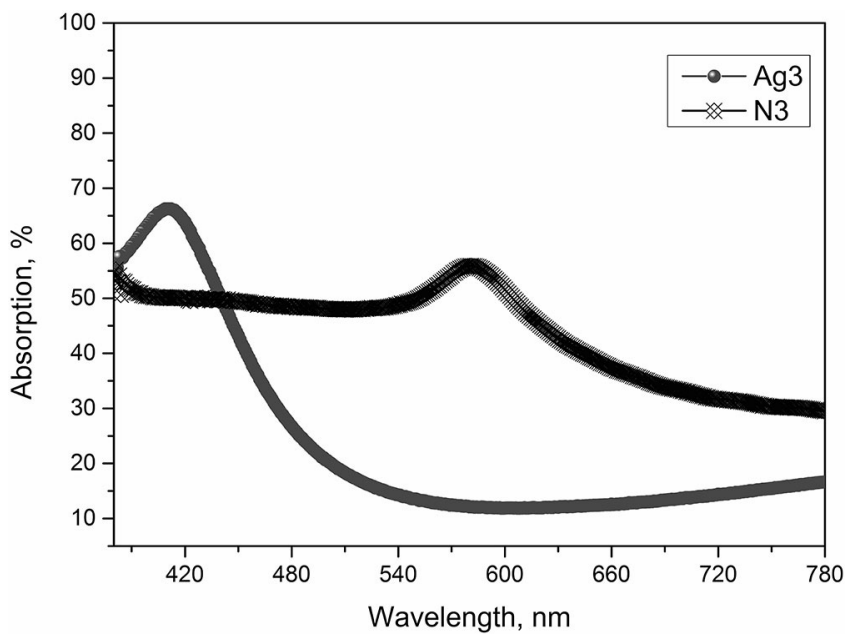

Figure 4. UV-visible absorption spectra of stained soda lime glasses vs. kind of stain (silver nitrate Ag3, copper nitrate N3). ure 5. For example, if we take a $4 \mu \mathrm{m}$ depth penetration reference of metallic nanoparticles into the glass, it is clearly observed that the silver content in the glass Ag3 is approximately $6 \%$ while for the same depth of penetration is about $2 \%$ and $0.5 \%$ of copper in glasses $\mathrm{N} 3$ and $\mathrm{S} 3$ respectively.

Nanoclusters density and volume according to the scanning electron microscope micrographs indicated a higher formation of that in soda lime glasses when silver is used, Figure 6. When copper is used, a few of nanoclusters are obtained. Then, it can be supposed that the structure of the soda lime glass manifests better with the formation of silver nanoparticles than with copper ones.

Sequential ion exchange glasses are characterized by absorption spectra with two peaks, Figure 7 . The first appears at $420 \mathrm{~nm}$ and the second less apparent at around $570 \mathrm{~nm}$ corresponding respectively to the formation of silver and copper nanoparticles.

For the painted glasses color variation, it increases with the growing of the silver concentration in the

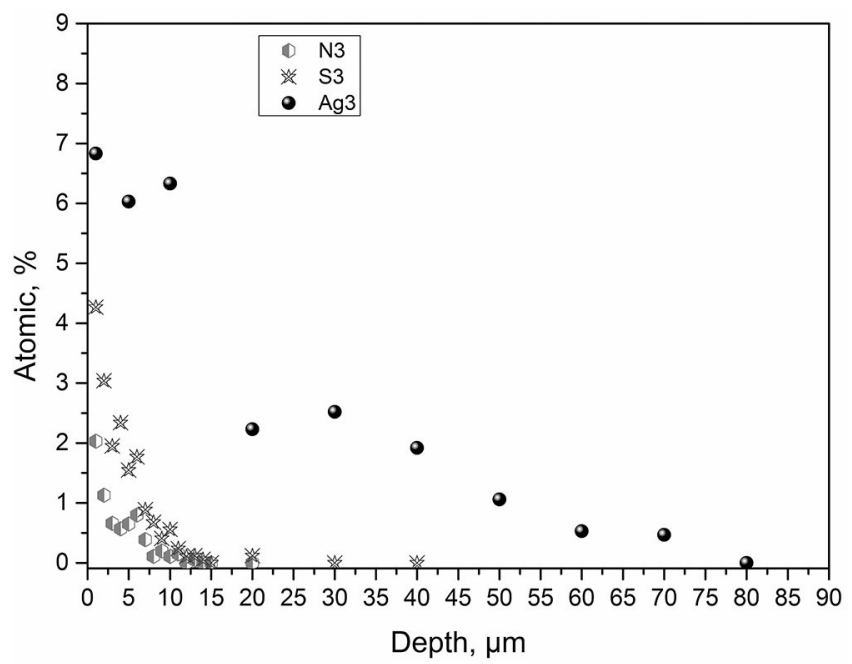

Figure 5. Concentration profiles of metallic nanoparticles in stained soda lime glass vs. stain kind.
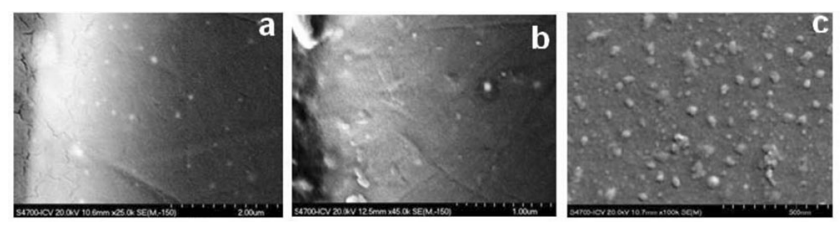

Figure 6. Micrographics obtained by scanning electronic microscope after ion-exchanged process for the soda lime glasses, a) copper nitrate stain N3, b) copper sulfate stain S3, c) silver nitrate stain Ag3. 


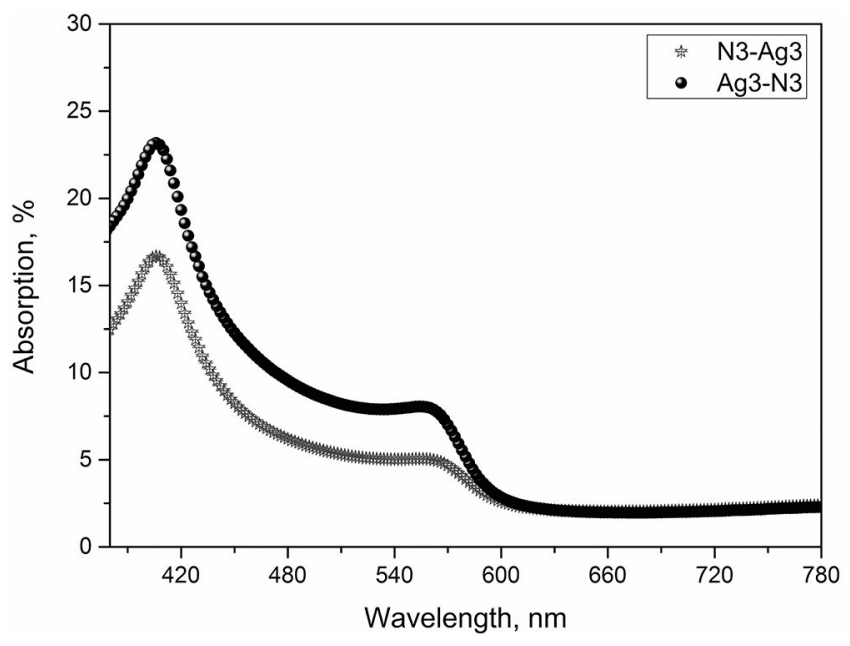

Figure 7. UV-visible absorption spectra of stained soda lime glasses vs. sequential ion exchange.

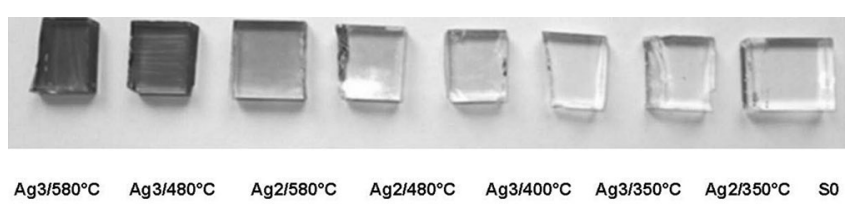

Figure 8. Coloration of painted glasses vs. silver concentration and heat treatment temperature.

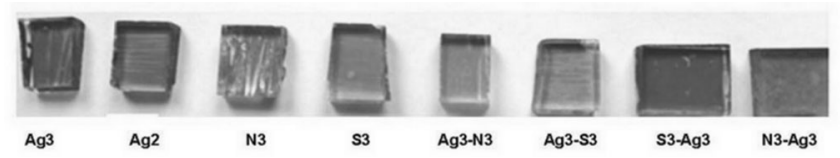

Figure 9. Coloration of painted glasses vs. mixed composition (heat treatment temperature is $580^{\circ} \mathrm{C}$ ). spread paste. In addition, opacity is observed when the treatment temperature is close to glass transition temperature $\left(T_{\mathrm{g}}\right)$. Moreover, a lack of ion exchange is observed at the treatment temperature about $350{ }^{\circ} \mathrm{C}$ and the glass remains colorless, Figure 8.

Moreover, colors obtained for painted glasses according to the spread paste composition demonstrate that the amber color is observed for glasses doped by silver while the red color is observed for those doped by copper, Figure 9. Concerning the sequential ionic exchange, the glasses react more with silver, consequently yellow and amber colorations are observed on the painted glasses.

The color parameters were calculated from the transmission spectra using the standards of the International Commission of Luminance (CIE) L*, a*, b* [20].

Values of these parameters depend on the concentration and composition of the mixture and the heat treatment temperature. Some glass surfaces doped with silver were yellow to brown amber color, and others were red when they are doped with copper, Table 2. On other hand, it was observed that glasses painted with copper could be colored only at $580{ }^{\circ} \mathrm{C}$. Otherwise, for the same temperature, the higher color intensity occurs for glasses doped with high concentration paste (Ag3). In summary, for the same concentration, the color is more intense when the treatment temperature is close to glass transition temperature $\left(T_{\mathrm{g}}\right)$. This confirms the existence of colorless glasses at $350{ }^{\circ} \mathrm{C}$, Figure 8, 9.

The radius of the nanoparticles "Rd" was calculated according to Doyle formula from the absorp-

Table 2. Optical appearance and chromatic factors of painted glasses*.

\begin{tabular}{llllllll}
\hline $\mathrm{T}$ & Chromatic factors & Ag3 & Ag2 & S3 & N3 & Ag3-N3 & N3-Ag3 \\
\hline $580{ }^{\circ} \mathrm{C}$ & $\mathrm{L}^{*}$ & 8.43 & 11.81 & 18.58 & 17.03 & 10.40 & 8.16 \\
& $\mathrm{a}^{*}$ & 20.99 & 01.25 & 24.55 & 23.18 & 18.30 & 24.27 \\
& $\mathrm{~b}^{*}$ & 27.01 & 20.28 & 18.41 & 17.42 & 24.50 & 26.70 \\
Color & & amber & yellow & red & red & amber & amber \\
$480{ }^{\circ} \mathrm{C}$ & $\mathrm{L}^{*}$ & 26.90 & 28.54 & 30.22 & 30.78 & & \\
& $\mathrm{a}^{*}$ & 19.03 & -1.46 & -2.19 & -2.02 & & \\
& $\mathrm{~b}^{*}$ & 27.27 & 12.67 & 4.29 & 2.86 & & \\
Color & & amber & pale yellow & incolor & incolor & & \\
$350{ }^{\circ} \mathrm{C}$ & $\mathrm{L}^{*}$ & 31.23 & 30.63 & 30.87 & 31.94 & & \\
& $\mathrm{a}^{*}$ & -0.89 & -0.93 & -0.74 & -0.81 & & \\
& $\mathrm{~b}^{*}$ & 0.68 & -0.54 & -0.45 & -0.27 & & \\
Color & & incolor & incolor & incolor & incolor & & \\
\hline
\end{tabular}

* $L^{*}$ : brightness, $a^{*}$ and $b^{*}$ : color coordinates. $a^{*}$ varies from negative to positive values (from green to red/magenta color). $b^{*}$ varies from negative to positive values (from blue to yellow color). 
tion spectra, Table $2[21,22]$. The $v_{\mathrm{f}}$ parameter represents the velocity of the electron in the mass of metal $\left(v_{\mathrm{f}}\right.$, for silver is $1.39 \times 10^{8} \mathrm{~cm} \mathrm{~s}^{-1}$ and copper is $\left.1.57 \times 10^{8} \mathrm{~cm} \cdot \mathrm{s}^{-1}\right)$, Equation $1[21,22]$. Some parameters can be obtained from the absorption spectra such: Width at half height of the absorption peak $(\Delta \lambda)$ which is related to the size of nanoclusters, $\lambda_{\mathrm{p}}$ $i$ s the characteristic wavelength at which the surface plasmon resonance (SPR) occurs.

$R d=\frac{V f \lambda p^{2}}{2 \pi c \Delta \lambda}$

Then, the absence of the absorption peak on the spectra, as in the glass painted with the lower concentration of copper nitrate or glasses treated at 350 ${ }^{\circ} \mathrm{C}$ produces colorless glasses. This means the absence of the metallic nanoparticles formation and only metallic ions were introduced into the glass.

The hardness values are the average of three nanoindentation tests performed on the surface of the painted glass, Table 3. The hardness of the raw glass is much higher than that of the painted glasses.

\section{Discussion}

The coloration of the glasses and the change of intensity are due, respectively, to the formation of the metal nanoparticles and the growth of the size and concentration of nanoclusters [23]. The formation of silver nanoparticles $\left(\mathrm{Ag}^{0}\right)$ or copper $\left(\mathrm{Cu}^{0}\right)$, due to the surface plasmon resonance (SPR), gives respectively a yellow or red color to glasses. This is reflected by the appearance on the optical absorption spectrums of a peak around $420 \mathrm{~nm}$ for the silver glasses or $570 \mathrm{~nm}$ for the copper ones.

The intensity and position of these peaks depend on the size and the concentration of copper or silver nanoparticles diffused in the glass [24]. It is noticed that the nanoparticles and nanoclusters size depend on the heat treatment temperature and the mixture spread concentration. The shifting of the surface plasmon resonance (SPR) peaks to the lowest wavelengths (toward blue), was caused by the increase in the heat treatment temperature and the concentration of the spread paste, which induce the nanoclusters size growth.

The sequential doping silver-copper $(\mathrm{Ag}-\mathrm{Cu})$ is characterized by the formation of silver nanoparticles over those of copper, identified by the existence of a highest intensity peak around $420 \mathrm{~nm}$ relatively to the $570 \mathrm{~nm}$ one.

Otherwise, copper-silver $(\mathrm{Cu}-\mathrm{Ag})$ doping behavior is different. Indeed, during the first step of doping (copper doping),copper nanoparticles are formed and when doping silver, copper nanoclusters dissolution can take place in the same time of formation of silver which induced again peak around $420 \mathrm{~nm}$ more intense than the $570 \mathrm{~nm}$ one [25].

Table 3. Essential characteristics of painted glasses.

\begin{tabular}{llllll}
\hline & $\Lambda p[\mathrm{~nm}]$ & $\Delta \lambda[\mathrm{nm}]$ & $\mathrm{Rd}[\mathrm{nm}]$ & Absorption [\%] & $\mathrm{H}[\mathrm{GPa}]$ \\
\hline N1 & - & - & - & 20 & - \\
N2 & 572 & 45 & 6.05 & 48 & - \\
N3 & 584 & 33 & 8.61 & 56 & $6.585 \pm 0.045$ \\
S1 & 584 & 40 & 7.10 & 63 & - \\
S2 & 574 & 35 & 7.84 & 68 & - \\
S3 & 569 & 30 & 8.99 & 78 & $6.410 \pm 0.040$ \\
N350 & - & - & - & 08 & - \\
N480 & 588 & 38 & 7.58 & 55 & - \\
N580 & 584 & 33 & 8.61 & 56 & $6.580 \pm 0.045$ \\
S350 & - & - & - & 12 & - \\
S480 & 575 & 35 & 7.87 & 55 & - \\
S580 & 569 & 30 & 4.99 & 78 & $6.410 \pm 0.040$ \\
Ag3 & 420 & 29 & 4.48 & 68 & $6.400 \pm 0.052$ \\
Ag-Cu & $410 / 566$ & $28 / 57$ & $4.02 / 3.80$ & $24 / 08$ & $6.480 \pm 0.062$ \\
Cu-Ag & $573 / 410$ & $68 / 32$ & - & $05 / 17$ & $6.560 \pm 0.055$ \\
S0 & - & - & & 08 & $6.750 \pm 0.025$ \\
\hline
\end{tabular}


The shift of indentations curves for the painted and raw glasses elucidate an alteration of the painted glass structure. The observed differences are less important when glasses were treated with copper nitrate. The hardness values of painted glasses induce the thesis that their structure underwent depolymerization, leading to the destruction of silicon-oxygen $(\mathrm{Si}-\mathrm{O})$ bonds. Consequently, the hardness of painted surfaces with silver and /or copper paste decreases.

\section{Conclusion}

The classic staining method was used for coloration of soda lime glasses and several characterizations were done. The obtained results allow the drawing of several conclusions. The formation of nanoparticles is reflected by the apparition of an absorption band at $420 \mathrm{~nm}$ and $570 \mathrm{~nm}$, respectively for silver and copper nanoparticles. The red color for copper and yellow or amber one for silver are obtained on the painted glasses. Annealing temperatures increase the band intensity of the absorption peak. The observed alteration of the painted glasses surfaces structure is proved by the shift between the indentation curves of the raw and painted glasses. The difference is less important in the case of glasses painted with copper nitrate. The formation and diffusion depth of silver nanoparticles is higher as those of copper nanoparticles. In summary, the studied glasses manifests better with silver nanoparticles formation and diffusion as with copper nanoparticles.

\section{References}

[1] A. Mezi, Phys. D, Toulouse University, France, 2013.

[2] G.A. Olah, Handbook of Nanostructured Materials and Nanotechnology, Academic Press, Burlington, USA, 2000.

[3] U. Kreibig, M. Vollmer, Optical properties of metal clusters, Springer series in Materials sciences, Berlin, Germany, 1995.

[4] P. Tartaj, M. Puerto-Morales, S. VentimillasVerdaguer, T. Gonzalez-Carreno, C.J. Serna, J. Phys. D, 2003, 36, 182.

[5] I. Sondi, B. Salopak-Sondi, J. Colloid Interface Sci., 2004, 275, 177.
[6] C. Gil, M.A. Villegas, Mater. Chem. Phys., 2004, 88, 185.

[7] A. Caiger-Smith, Luster Pottery Techniques, Tradition and Innovation in Islamic and Western world, Edited by Faber and Faber, London, UK, 1989.

[8] D. Richard, P. Roussignol, C. Flytzanis, Opt. Lett., 1985, 10, 511.

[9] F. Gonella, A. Quaranta, E. Cattaruzza, S. Padovani, C. Sada, F.D. Acapito, C. Maurizio, Comput. Mater. Sci., 2005, 33, 31.

[10] A.L. Stepanov, D.E. Hole, P.D. Towsend, J. Non-Cryst. Solids, 1999, 260, 65.

[11] J. Zhang, J. Sheng, Int. J. Hydrogen Energy, 2009, 34, 3531.

[12] G. De, A. Licciulli, C. Massaro, L. Tapfer, M. Catalano, G. Battgin, C. Meneghini, P. Mazzoldi, J. Non-Cryst. Solids, 1996, 194, 225.

[13] Y. Dimitriev, Y. Ivanova, A.E.D. Mohamed Tawfik, M. Gancheva, R. Iordanova, J. Univ. Bombay Sci., 2012, 47, 415.

[14] P.W. Wang, L. Zhang, J. Am. Ceram. Soc., 1997, 80, 2285.

[15] F. Rubio, S. Pérez-Villar, M.A. Garrido, J. Rubio, J.L. Oteo, J. Nanopart. Res., 2009, 8, 89.

[16] P. Le Vieil, The Art of Painting on Glass and Glazing, Edited by Delatour, Paris, France, 1774.

[17] W.A. Weyl, Colored Glasses, Edited by Dawson's of Pall Mall, London, 1959.

[18] A. Chorfa, N. Belkhir, F. Rubio, J. Rubio, Ceram.-Silik., 2012, 56, 69.

[19] W.C. Oliver, G.M. Pharr, J. Mater. Res., 1992, 7, 156.

[20] C. Huang, A.N. Cormack, J. Chem. Phys., 1990, 93, 8180 .

[21] S. Pérez-Villar, J. Rubio, J.L. Oteo, J. NonCryst. Solids, 2008, 354, 1833.

[22] W.T. Doyle, Phys. Rev. B, 1989, 39, 9852.

[23] A.Y. Zhang, T. Suetsugu, K. Kadono, J. NonCryst. Solids, 2007, 353, 44.

[24] D.E. Hole, A.L. Stepanov, P.D. Townsend, Nucl. Instrum. Methods Phys. Res. Sect. B, 1999, 148, 1054.

[25] D. Manikandan, S. Mohan, P. Magudapathy, K.G.M. Nair, Physica. B: Condensed Matter, 2003, 325, 86.

Received in final form: October $29^{\text {th }} 2017$ 